\title{
Имитационный подход к моделированию лавинного пробоя p-n-перехода
}

\author{
(C) А.C. Шашкина ${ }^{1}$, С.Д. Ханин ${ }^{2}$ \\ ${ }^{1}$ Санкт-Петербургский государственный институт кино и телевидения, \\ 191119 Санкт-Петербург, Россия \\ ${ }^{2}$ Военная академия связи им. С.М. Будённого, \\ 194064 Санкт-Петербург, Россия \\ E-mail: shashkinaas@yandex.ru
}

Поступила в Редакцию 29 октября 2018 г.

В окончательной редакции 25 января 2019 г.

Принята к публикации 30 января 2019 г.

Проведено экспериментальное исследование лавинного пробоя $p-n$-перехода с целью изучения временно́го распределения микроплазменных импульсов. Выявлено, что наблюдаемый вид микроплазменного шума не описывается существующей моделью процессов, происходящих при лавинном пробое $p-n$-перехода. С помощью имитационного моделирования была разработана компьютерная модель, проясняющая механизмы неустойчивости микроплазмы и учитывающая электрические и температурные зависимости лавинного пробоя, которая согласуется с результатами экспериментов.

DOI: 10.21883/FTP.2019.06.47741.8983

\section{1. Введение}

Лавинный пробой реальных полупроводниковых структур сильно локализован и имеет микроплазменный характер. Локализация пробоя обычно происходит в местах различного рода дефектов. Это могут быть вакансии, дислокации, включения второй фазы, неоднородности в распределении легирующих примесей, наличие амфотерных примесей. Вблизи дислокации имеются области сжатия и расширения кристаллической решетки, что влияет на ширину запрещенной зоны. Помимо этого, дислокации являются центрами осаждения примесей и вдоль них повышены коэффициенты диффузии примесей. Значит, вероятность того, что в области дефекта окажется порождающий лавину носитель, выше, чем для бездефектной области. Кроме того, концентрация носителей заряда в этом месте выше, а эффективная толщина обедненного слоя ниже. Локально повышается напряженность поля, уменьшается напряжение пробоя, образуются микроплазмы. Ток, протекающий через каждую микроплазму, носит характер коротких импульсов, появляющихся и исчезающих в случайные моменты времени $[1,2]$.

Кроме того, важной причиной образования микроплазм могут являться включения второй фазы, которые искажают распределение электрического поля в области пространственного заряда (ОП3) $p$-n-перехода [1,3]. Однако законченной теории образования и функционирования микроплазменного пробоя до сих пор не существует.

Наиболее значимыми причинами прерывания лавинного тока являются:

- саморазогрев микроплазмы, приводящий к уменьшению длины свободного пробега, к увеличению концентрации свободных носителей, к увеличению количества дефектов кристаллической решетки и их скоплений;
- запирающее электрическое поле, которое в одних случаях может усиливаться, а в других - ослабляться, что также влияет на концентрацию носителей и их энергию;

- местоположение дефектов и их скоплений из-за того, что лавинный пробой сильно локализован и микроплазмы чаще всего возникают в местах структурных несовершенств и их скоплений.

В настоящее время преобладающей считается гипотеза, согласно которой вероятность выключения микроплазмы определяется саморазогревом структуры.

Носители в область микроплазмы могут поставляться путем $[1,4]$ :

1) диффузии из базовых областей;

2) термогенерации носителей в ОПЗ;

3) туннельного эффекта;

4) реэмиссии с ловушек носителей, захваченных в период протекания предшествующего импульса тока;

5) эмиссии носителей заряда с глубоких центров (ГЦ) $[3,5,6]$.

Известно, что для получения красного свечения фосфид галлия $(\mathrm{GaP})$ легируют цинком и кислородом. Кислород, введенный в $\mathrm{GaP}$, не является ни мелким донором, ни акцептором, но создает глубокий донорный уровень [7,8].

В работах $[2,9,10]$ приведены результаты экспериментальных исследований обратносмещенных GaP-светодиодов и показано, что временно́е распределение микроплазменных импульсов имеет специфический вид. Оно является случайным и в то же время обладает свойством самоподобия. По характеру спектра данный сигнал нельзя отнести ни к белому, ни к розовому шуму.

Также подвергнут анализу спектр шума лавинообразования, аналитическое выражение для которого было предложено А.С. Тагером. Показано, что существующая 
модель процессов, происходящих при лавинном пробое полупроводниковых приборов, плохо совпадает с результатами экспериментов.

Следовательно, необходимо предложить и обосновать методы исследования, которые помогут объяснить природу специфики сигнала и дополнить теорию ударной ионизации.

\section{2. Методика эксперимента}

Основными объектами для исследований были выбраны фосфидгаллиевые светодиоды красного излучения АЛ102АМ ( $\mathrm{GaP}: \mathrm{ZnO})$ в количестве 300 штук. Ha GaP-светодиоды подавалось постоянное обратное напряжение (напряжение питания) от 10 до 19 В. Сигнал, получаемый на нагрузке светодиода, усиливался и поступал на аналого-цифровой преобразователь (АЦП). Дальнейшая обработка сигнала с АЦП осуществлялась с помощью математического пакета Matlab. Программным путем можно также управлять напряжением, подаваемым на светодиод.

Над светодиодом размещался микроскоп с видеокамерой, позволяющий в режиме реального времени на экране монитора наблюдать за светящимися областями на кристалле светодиода. Также имелась возможность видеозаписи.

Методика эксперимента заключалась в следующем. Напряжение питания постепенно увеличивалось с шагом $\sim 0.005$ В до тех пор, пока не появлялась первая микроплазма. Скорость роста напряжения снижалась, но напряжение продолжали увеличивать до появления следующей микроплазмы. Процесс продолжается до тех пор, пока через светодиод не начинает течь постоянный обратный ток. При этом производится запись сигнала в память компьютера и его отображение в реальном времени на экране в окне Matlab. Существует возможность произвести запись повторно: либо удалив предыдущую, либо поверх существующей. Следуя предложенной методике, были записаны данные для 300 светодиодов.

На основании этих данных для каждого светодиода строилась вольт-амперная характеристика (BAX) зависимость обратного тока через светодиод от обратного напряжения в вольтах, подаваемого на светодиод. С помощью специально разработанного программного обеспечения для каждой точки BAX можно было посмотреть осциллограмму импульсного тока через светодиод - зависимость напряжения, падающего на нагрузке светодиода от времени.

\section{3. Результаты эксперимента. Основные идеи исследования}

Были построены кривые фрактальной размерности и рассчитана фрактальная размерность записанных сигналов. Ее величина существенно зависит от приложенного к светодиоду обратного напряжения. По анализу формы импульсов микроплазменного тока и численных различий фрактальных размерностей была разработана классификация типов лавинного пробоя.

Наличие фрактальных свойств (свойств самоподобия) и разноуровневости тока указывает на то, что при лавинном пробое одновременно действуют несколько противоборствующих факторов, и при анализе механизмов лавинного пробоя $p-n$-перехода их необходимо рассматривать в совокупности. Следует учитывать максимально возможное количество факторов и зависимостей, учитывать зависимость и концентрации, и подвижности, и длины свободного пробега, и от температуры, и от запирающего поля.

Для моделирования лавинного пробоя $p-n$-перехода был применен системный подход, следствием которого стало представление $p-n$-перехода в виде системы с большим количеством положительных, т.е. способствующих лавинному пробою, и отрицательных, т.е. подавлящих пробой, обратных связей.

Также важным подходом к исследованию является отказ от работы с конкретными числовыми значениями, а оперирование плотностями распределения вероятностей.

\section{4. Средства исследования}

Реализацию подхода, описанного выше, обеспечивало имитационное компьютерное моделирование.

В основе любого моделирования лежит понимание физической сущности моделируемых процессов. Затем на его основе разрабатывается математическая модель, обычно с помощью систем дифференциальных уравнений. Используя какой-либо математический пакет, можно решить эту систему дифференциальных уравнений и разработать компьютерную модель. В этом состоит суть аналитического компьютерного моделирования. А именно изучаются математические модели реального объекта в виде алгебраических, дифференциальных и других уравнений, а также предусматривающих осуществление однозначной вычислительной процедуры, приводящей к их точному решению.

В основе имитационного моделирования также лежит математическое описание. Однако применяется оно несколько по-другому. При имитационном моделировании исследуются математические модели в виде алгоритмов, воспроизводящих функционирование исследуемой системы путем последовательного выполнения большого количества элементарных операций $[11,12]$.

Имитационный подход позволяет рассмотреть любую гипотезу функционирования системы и максимально использовать всю имеющуюся у исследователя информацию о системе [13].

Следует заметить, что индивидуальные характеристики конкретных электронов принципиально не могут быть учтены в аналитической модели и подлежат только имитационному моделированию. 


\section{5. Физические основы имитационного моделирования}

Для того чтобы произошла ударная ионизация, необходимо, чтобы электрон при соударении с атомом кристаллической решетки имел энергию не менее ширины запрещенной зоны $E_{g}$. Ширина запрещенной зоны фосфида галлия составляет 2.27 эВ при температуре $300 \mathrm{~K}$. Пороговое значение энергии ионизации чаще всего принимают равным $1.5 E_{g}$. Но необходимо учитывать, что существуют и „подпороговые“ механизмы ударной ионизации. Следовательно, первой величиной, определяющей вероятность $p_{1}$ того, что произойдет ударная ионизация, является энергия взаимодействующего электрона.

Существует несколько вариантов того, каким образом электрон наберет необходимую для ударной ионизации энергию.

При рассмотрении поведения полупроводников в сильных полях учитывают три механизма рассеяния [1]: упругое рассеяние на акустических фононах, неупругое рассение на оптических фононах и ударную ионизацию. В электрических полях напряженностью более $10^{3} \mathrm{~B} / \mathrm{cm}$ преобладающим механизмом становится рассеяние на оптических фононах, а рассеянием на акустических фононах можно пренебречь.

Электрон ускоряется внешним полем. При соударении с атомом он теряет часть своей энергии - энергию рамановского фонона. Величина этой энергии всегда одинакова и для фосфида галлия составляет 0.05 эВ. Если энергия электрона превышает энергию оптического фонона, то потеряв часть своей энергии он продолжит свое движение до следующего соударения. Так будет продолжаться до тех пор, пока энергия электрона не станет достаточной для того, чтобы при соударении вышибить пару электрон-дырка. В этом случае начнет действовать новый механизм рассеяния - ударная ионизация. Необходимым условием при таком механизме является: электрон на длине свободного пробега должен набрать энергию больше энергии оптического фонона, т.е. более 0.05 эВ. В этом случае за несколько этапов он может набрать энергию, достаточную для ионизации. Для обозначения пути, пройденного электроном, введем термин „Длина разгона“, включающий в себя несколько длин свободного пробега электрона.

В очень сильных полях существует возможность того, что электрон наберет энергию более 2.27 эВ и на одной длине свободного пробега.

Необходимо учитывать, что длина свободного пробега $L$ в кристалле не является постоянной строго определенной величиной. Средняя длина свободного пробега в фосфиде галлия принимается равной $35 \AA$. Однако в реальном кристалле она может быть намного больше или намного меньше. Кроме того, длина свободного пробега значительно меняется с температурой.

Вероятность акта рассеяния частиц связана с сечением рассеяния частиц [14].

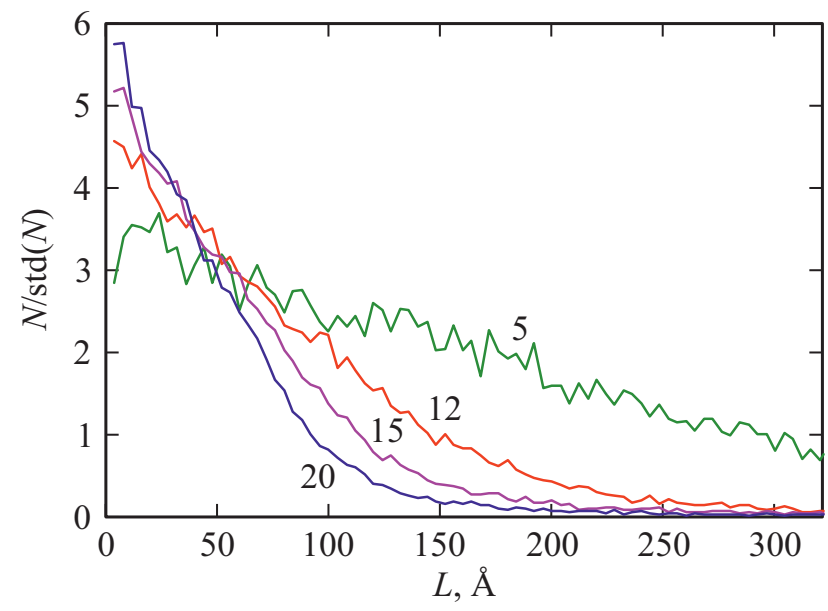

Рис. 1. Гистограмма распределения длин свободного пробега при различных $\sigma$.

При моделировании мы берем за основу кристаллическую решетку фосфида галлия. Тип решетки: кубическая гранецентрированная. Постоянная решетки $a=0.545$ нм.

При моделировании имитировалось движение электронов в кристалле. Электроны двигались по ОПЗ, количество электронов, используемых при одной имитации, - 10 тысяч. Если электрон столкнулся с атомом, то считалось расстояние, которое он прошел от предыдущего столкновения, т.е. расстояние между столкновениями - длина свободного пробега $L$. Электрон движется дальше, снова сталкивается с атомом, снова считается длина свободного пробега. Таким образом набирались сотни тысяч длин свободного пробега. Программа запускалась несколько раз, при этом перебирались значения сечения рассеяния $\sigma$ диапазона от $10^{-19}$ до $10^{-18} \mathrm{~cm}^{2}$. На основании этого опыта была построена гистограмма распределения длин свободного пробега $L$ при различных $\sigma$ (рис. 1 ). По оси абсцисс отложены значения $L$ в $\AA$. По оси ординат - нормированные (деленные на дисперсию) значения количества $N$ гистограммы длин пробегов с шагом в $4 \AA$. На рисунке показаны кривые для четырех значений сечения рассеяния, умноженного на $10^{-19} \mathrm{~cm}^{2}$.

Сечение рассеяния $\sigma$ определяет вероятность $p_{1}$ возникновения лавины в потенциальной области пробоя, сопряженной с дефектом (микроплазмы), в течение 1 пкс. Эта вероятность сильно зависит от местоположения дефекта, и в модели $p_{1}$ есть произведение:

$$
p_{1}=p_{a 1} \cdot p_{b 1},
$$

где $p_{a 1}-$ вероятность присутствия электрона в потенциальной области пробоя, $p_{b 1}-$ вероятность акта ударной ионизации.

Вероятность $p_{a 1}$ определяется исходя из размеров дефекта и концентрации электронов в ОПЗ. 
Роль дефекта при лавинном пробое сводится к следующему:

- на дефекте может сработать „подпороговый“ механизм ударной ионизации: за счет искривления электрического поля образование электронно-дырочной пары произойдет при энергиях, меньших пороговой энергии ионизации для остальной части кристалла;

— дефект кристаллической решетки изменяет длину разгона носителя.

Вероятность $p_{b 1}$ определяется как отношение числа электронов $n$, которые при столкновении с атомом вышибли пару электрон-дырка, к общему числу электронов $m$ :

$$
p_{b 1}=\frac{n}{m} \text {. }
$$

Вероятность ударной ионизации $p_{b 1}$ зависит от длины разгона электрона и энергии ионизации. С помощью модели была построена зависимость вероятности ударной ионизации $p_{b 1}$ от напряжения пробоя $U$ при различных энергиях ионизации. Она представлена на рис. 2. Значения энергии ионизации подписаны рядом с кривыми. Для того чтобы учесть „подпороговый“ механизм ударной ионизации, для дальнейшей работы модели было выбрано значение энергии ионизации равное 2.9 эВ.

Диапазон вариаций энергии ионизации для фосфида галлия обусловлен одновременным выполнением в процессе ионизации законов сохранения энергии и импульса и, следовательно, выражением [15]

$$
E_{\min }=\frac{2 m_{n}+m_{p}}{m_{n}+m_{p}} E_{g}
$$

где $m_{n}, m_{p}$ - изотропные эффективные массы электронов и дырок соответственно. Отсюда следует, что в зависимости от соотношения между массами минимальная энергия ионизации изменяется от $E_{g}$ до $2 E_{g}$ [1]. Часто значение пороговой энергии ионизации принимается равным $1.5 E_{g}$.

Вероятность развития лавины $p_{2}$ есть вероятность возникновения лавины в потенциальной области пробоя, сопряженной с дефектом (микроплазмы), в течение 1 пкс после начала процесса лавинообразования. Вероятность $p_{2}$ определяет поддержание тока через микроплазму. По аналогии с вероятностью $p_{1}$ вероятность $p_{2}$ равна произведению:

$$
p_{2}=p_{a 2} \cdot p_{b 2},
$$

где $p_{a 2}-$ вероятность присутствия электрона в микроплазме, $p_{b 2}-$ вероятность акта ударной ионизации после начала процесса лавинообразования.

Первоначально в результате естественной диффузии электронов и дырок вблизи $p-n$-перехода образуется запирающее электрическое поле, область пространственного заряда неподвижных ионов. При подаче напряжения в прямом направлении приложенное поле совпадает по направлению с основными носителями и уменьшает запирающий слой.

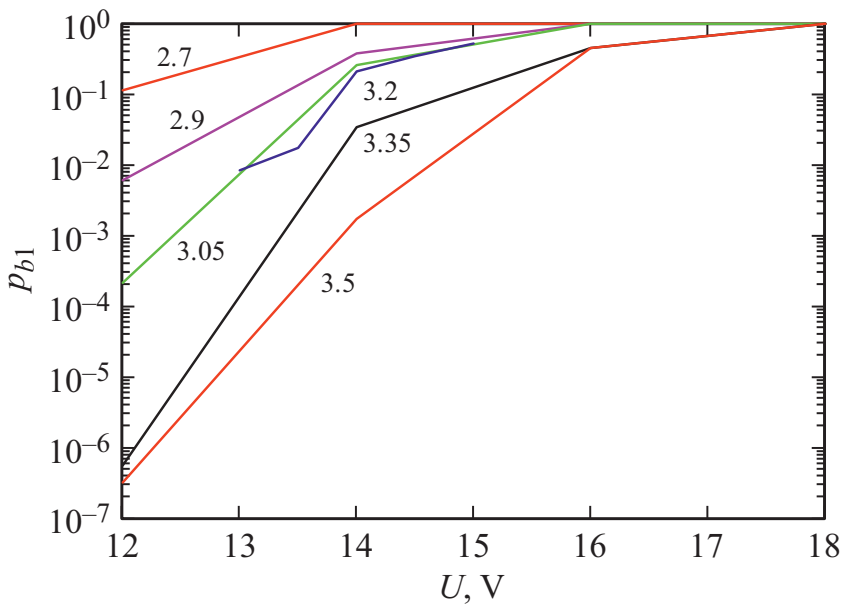

Рис. 2. Зависимость вероятности ударной ионизации от напряжения пробоя при различных энергиях ионизации.

В нашем случае, когда напряжение подается в обратном направлении, направление внешнего поля совпадает с запирающим полем, тем самым усиливая его, и поле становится разгоняющим. Это означает, что неосновному носителю (электрону) легче разогнаться до энергии, достаточной для того, чтобы при соударении с неподвижными атомами решетки выбить электроннодырочную пару. Наиболее сильный разгон происходит в середине обедненной зоны. Разогнавшись, электроны свободно пролетают в родную для себя $n$-область, по которой растекаются. В свою очередь с другой стороны $p-n$-перехода скапливаются дырки. Скапливаясь на стороне положительно заряженных ионов, они усиливают разгоняющее поле. Это короткая положительная обратная связь. Вероятность $p_{b 2}$ увеличивается.

Далее дырки начинают дрейфовать к отрицательным ионам с меньшей, чем электроны к положительным ионам, скоростью. Подвижности электронов и дырок в фосфиде галлия соответственно равны: $\mu_{e}=0.019 \mathrm{~m}^{2} /(\mathrm{B} \cdot \mathrm{c})$ и $\mu_{p}=0.012 \mathrm{~m}^{2} /(\mathrm{B} \cdot \mathrm{c})$ [16]. Тем самым перемещающееся облако дырок начинает ослаблять разгоняющее поле. Эта отрицательная обратная связь уже будет длиннее, чем положительная.

Однако, несмотря на временно́е ослабление разгоняющего поля, облако дырок, приближаясь к противоположным границам ОПЗ, к $p$-области, в то же время начинает уменьшать тормозящее поле на границе $p$-область-ОПЗ и оттягивать на себя электроны. Преодолев тормозящее поле, электронам легче попасть в область дефекта. При создавшихся условиях электроны могут туннелировать и с ловушек. Тем самым там повышается концентрация электронов и, следовательно, увеличивается вероятность $p_{a 2}$ обнаружения электрона.

Таким образом, движение вторичных дырок, которые то ослабляют, то усиливают запирающее поле, оказывает сильное влияние на величину вероятности $p_{2}$. 
Также на величину вероятности $p_{2}$ существенное влияние оказывает фактор саморазогрева. Но по сравнению с движением носителей зарядов этот процесс является медленным и обеспечивает макроскопическую, т. е. медленную обратную связь.

Основными факторами саморазогрева микроплазмы являются:

1) уменьшение длины разгона носителя, т.е. его подвижности;

2) увеличение концентрации электронов.

Первый фактор может привести к прекращению лавинного пробоя за счет того, что чем меньше длина разгона носителя, тем меньше шансов у него набрать достаточную для ионизации энергию. Следовательно, в этом случае вероятность $p_{b 2}$ развития лавины уменьшается.

Второй же фактор приводит к увеличению вероятности наличия электрона в области дефекта $p_{a 2}$. Таким образом, изменение температуры $p-n$-перехода вблизи области пробоя дает нам 2 противоборствующих фактора.

Видно, что вероятность $p_{2}$ зависит и от состояния системы на макроуровне. Макропараметры меняются значительно медленнее микропараметров, поэтому вероятность возникновения лавины становится гораздо меньше, чем вероятность ее развития, т.е. $p_{1} \ll p_{2}$.

При значении энергии ионизации, равном 2.9 эВ, было построено семейство кривых зависимости вероятности $p_{b 1}$ от приложенного напряжения при различных значениях температуры $\Delta T$. Аппроксимация этих кривых проведена логистической функцией вида:

$$
f(x)=\frac{1}{1+a \cdot \exp (x+b)},
$$

где $a$ и $b-$ параметры, подбираемые в автоматическом или ручном режиме.

Моделирование и расчет теплопередачи производится на трехмерной сетке, размер которой соответствует предполагаемому размеру микроплазмы. Шаг сетки равен $l$, площадь грани одного „кубика“, образованного сеткой, равна $S$. Также задается плотность вещества $\rho$. Исходя из средней интенсивности импульса тока и его длительности мы можем определить количество электронов, участвующих в пробое, а значит, и потерю энергии на нагревание $Q$. Количество теплоты, выделяющееся в одном „кубике“, равно

$$
Q=c \cdot m \cdot \Delta T,
$$

где $c$ - удельная теплоемкость, $m=\rho V=\rho S l-$ масса „кубика“; $\Delta T$ - изменение температуры. Отсюда, зная $Q$, можно определить температуру:

$$
T=\frac{\Delta Q}{c \rho S l}
$$

В интегральной форме закон теплопроводности имеет вид

$$
P=\frac{\lambda S}{l} \Delta T
$$

где $P$ - мощность тепловых потерь, $\lambda-$ коэффициент теплопроводности. Таким образом, суммарное количество теплоты, переданное „кубику“ с номером $i, j, k$ от соседних „кубиков““ за единицу времени, будет равно

$$
\begin{aligned}
\sum Q_{i, j, k}= & \frac{\lambda S}{l} \Delta t\left(\Delta T_{i-1, j, k}+\Delta T_{i+1, j, k}+\Delta T_{i, j-1, k}\right. \\
& \left.+\Delta T_{i, j+1, k}+\Delta T_{i, j, k-1}+\Delta T_{i, j, k+1}\right) .
\end{aligned}
$$

С учетом того что температура самого „кубика“ нам уже известна, упрощаем выражение:

$$
\begin{aligned}
\sum Q_{i, j, k}= & \frac{\lambda S}{l} \Delta t\left(6 T_{i, j, k}-T_{i-1, j, k}-T_{i, j-1, k}\right. \\
& \left.-T_{i, j+1, k}-T_{i, j, k-1}-T_{i, j, k+1}\right) .
\end{aligned}
$$

\section{6. Заключение}

Для моделирования лавинного пробоя $p-n$-перехода был применен системный подход, следствием которого стало представление $p-n$-перехода в виде системы с большим количеством положительных, т.е. способствующих лавинному пробою, и отрицательных, т. е. подавлящих пробой, обратных связей. В модели поддержание и прекращение пробоя определяется путем непрерывного вычисления вероятностей $p_{1}$ и $p_{2}$ при меняющихся параметрах системы.

По итогам проделанной работы было продемонстрировано, что именно наличие нескольких нелинейных обратных связей и оперирование характеристиками конкретных электронов в имитационной модели позволяют получить сигнал, сходный с тем, что был получен экспериментально. Анализировались осциллограммы полученных сигналов, а также степень их фрактальности $[9,10]$.

\section{Список литературы}

[1] И.В. Грехов, Ю.Н. Сережкин. Лавинный пробой $p-n-$ перехода в полупроводниках (Л., Энергия, Ленингр. отдние, 1980) с. 80.

[2] М.В. Воротков, Н.Н. Скворцов, А.С. Шашкина. Матер. III Всерос. науч.-практ. конф. „Инновационные технологии в медиаобразовании“ (СПб., СПбГИКиТ, 2015) вып. 3, с. 65.

[3] В.К. Ионычев. Автореф. канд. дис. (Мордовский гос. ун-т им. Н.П. Огарева, 1999).

[4] R.H. Haitz. J. Appl. Phys., 36, 3123 (1965).

[5] С.В. Булярский, Ю.Н. Сережкин, В.К. Ионычев. ФТП, 33, 1345 (1999).

[6] В.К. Ионычев, А.Н. Ребров. ФТП, 43, 980 (2009).

[7] А.Э. Юнович. Излучательная рекомбинация в полупроводниках (М., Наука, 1972) с. 222. 
[8] F. Schubert. Light-emitting diodes. 2nd edn (Cambridge, Cambridge University Press, 2006).

[9] А.С. Шашкина, А.В. Кривошейкин, Н.Н. Скворцов, М.В. Воротков. Науч.-техн. вестн. информационных технологий, механики и оптики, 16, 864 (2016).

[10] А.С. Шашкина, А.В. Кривошейкин, Н.Н. Скворцов, М.В. Воротков. Науч.-техн. ведомости СПбГПУ. Физ.-мат. наук, 253 (4), 85 (2016).

[11] В.Н. Ашихмин, М.Б. Гитман, И.Э. Келлер, О.Б. Наймарк, В.Ю. Столбов, П.В. Трусов, П.К. Фрик. Введение в математическое моделирование: учебное пособие (М., Логос, 2005).

[12] Е.M. Кудрявцев. GPSS World. Основы имитационного моделирования различных систем (М., ДМК Пресс, 2004).

[13] А.Б. Горстко. Познакомьтесь с математическим моделированием (М., Знание, 1991).

[14] Ш.М. Коган. Электронный шум и флуктуации в твердых mелах (М.,Физматлит, 2009).

[15] Дж. Кэррол. СВЧ-генераторы на горячих электронах (М., Мир, 1972).

[16] В.В. Пасынков, В.С. Сорокин. Материалы электронной техники (СПб., Лань, 2001).

Редактор Г.А. Оганесян

\title{
The simulation approach to modeling avalanche breakdown of the $p-n$-junction
}

\author{
A.S. Shashkina ${ }^{1}$, S.D. Hanin ${ }^{2}$ \\ ${ }^{1}$ St. Petersburg University of Film and Television, \\ 191119 St. Petersburg, Russia \\ ${ }^{2}$ Military Academy of Communications S.M. Budyonny, \\ 194064 St. Petersburg, Russia
}

Abstract An experimental study of the avalanche breakdown of the $p-n$-junction was carried out in order to study the temporal distribution of microplasma pulses. It was revealed that the observed appearance microplasma noise is not described in the existing model processes that occur during avalanche breakdown of $p-n$-junction. Using simulation, a computer model was developed that clarifies the mechanisms of microplasma instability and takes into account the electrical and temperature dependences of avalanche breakdown, which is consistent with the results of experiments. 